\title{
Genetic differentiation of quantitative characters between populations or species II: optimal selection in infinite populations
}

\author{
Prem Narain* and \\ Ranajit Chakraborty
}

Centre for Demographic and Population Genetics, University of Texas Health Science Center, Houston, Texas, U.S.A.

\begin{abstract}
Using a new and more general genetic model called the discrete-allelic state model and assuming discrete-time process, the evolutionary changes of genetic variation of quantitative characters, controlled by a few loci, within and between populations during the process of genetic differentiation of populations or species, are studied under the effects of mutation and centripetal selection in infinitely large populations. While in a finite population and ignoring selection, the rate of change of additive genetic variance depends on mutation and effective population size, traits under optimal selection in infinitely large populations go through the dynamics of a rather complicated form depending on the relative intensities of selection and mutation. When a population, which has reached steady-state by mutation-selection balance, splits into two, in one of which the same optimum genotype holds but in the other the optimum shifts a few standard deviations away from the original optimum, the corresponding daughter population starts differentiating from its sister population by favouring certain class of mutant alleles and discarding others which were originally favoured. During this process of turn over of genes, both the intra- and inter-populational variances undergo a complicated change, and the ratio of the former to the latter is a non-linear function of time of divergence. This pattern is qualitatively very different from the case when selection is absent. The intra-population distribution of genotypic values, during this transition, is shown to deviate considerably from normality. The presence of linkage seems to retard the accumulation of intra-populational genetic variance. The implications of these results are discussed in comparison with the earlier findings of evolutionary models of quantitative traits.
\end{abstract}

\section{INTRODUCTION}

The early attempts to explain genetic variability of quantitative characters made by Fisher (1922), Wright (1931, 1937), Haldane (1932), Robertson (1956) and Latter (1960) dealt with models based on multiple loci but each with only two segregating alleles. Modern understanding of molecular genetics, however, indicate that a gene is subdivisible into a very large number of variable nucleotide sites so that a model involving segregation of infinitely many alleles at a given locus is more realistic than the early attempts. Crow and Kimura (1964) introduced, for the first time, an infinite allele model which was later studied by Kimura (1965) in detail. In this study, mutation was considered as producing multiple alleles with varying

\footnotetext{
*Present address: Indian Agricultural Statistics Research
} Institute, Library Avenue, New Delhi 110 012, India. phenotypic effects in a continuous manner at each of the several loci involved in the inheritance of quantitative characters. Later on, several workers such as Latter and Novitski (1969), Latter (1970), Slatkin (1970), Lande (1975, 1976, 1977, 1979, 1980), Cavalli-Sforza and Feldman (1976), Bulmer (1972, 1980), Fleming (1979), Karlin (1979) studied the evolution of quantitative characters incorporating the effects of mutation, selection and genetic drift either singly or jointly. More recently, Nagylaki (1984), Turelli (1984, 1985), Gillespie (1984) and Gimelfarb (1986) have addressed similar problems.

In these studies (with the exception of Latter, 1970, Lande, 1975 and Gillespie, 1984), the primary concern was to explain the magnitude of genetic variance within a population and little consideration was given to the genetic differentiation between population or species. Moreover, none of these workers adopted a more realistic 
model of mutation involving discrete change of state. Chakraborty and Nei (1982) developed therefore a new mutation model called "discrete allelic effect" to examine the extent of genetic variation of a quantitative trait within a population as well as the same between two populations during the process of their genetic differentiation. But their study considered the forces of mutation and random drift only and selection was ignored.

As mentioned above, such a theory needs further revision as most quantitative characters are subject to adaptive changes and hence the role of selection cannot be ignored. Even though such adaptive genetic change is often triggered by environmental changes, as observed in the temporal changes in the cephalic index of Japanese skulls over the past 500 years (Suzuki, 1960), in certain characters the effect of environmental factors seems to be relatively simple. For example, skin pigmentation in man is to a great extent related to adaptation to sunlight, so that by knowing the sunlight intensity for any population, we may be able to model the evolutionary change of pigmentation. Thus, by a joint consideration of selection, mutation and random genetic drift, we may determine the selective stress due to adaptation for a changed environment during the period of human migration. In general, however, a rigorous analysis of joint effects of all the three factors viz. selection, mutation and random drift seems to be very complex.

The evolution of quantitative characters can be studied in either of the two ways. One way would be to define and study the underlying models at the level of phenotype avoiding any reference to gene frequencies. The other way could be to define and study a genetic model assuming that a complete genetic analysis of the traits is possible. In the latter case, one has to start from the simplest situation of a single locus with two alleles and build over it the more complex systems of infinitely many alleles at a locus, several loci, linkage and epistatic effects etc. The results obtained from the simpler situations of one or two loci give an insight to the problem, particularly for characters such as skin pigmentation in man which is believed to be controlled by a few loci say 5 to 6 with major effect. The rate of evolution, for such characters, is fairly rapid and modelling with few loci would be realistic.

In this paper, we follow the latter approach and study the evolutionary changes of genetic variance within and between populations for quantitative characters determined by a few loci with major effects such as skin pigmentation. For this purpose we use the discrete-time, discrete allelic-state model with mutation and random drift. The majority of the discussions of this paper involves mutation and selection in an infinitely large population, and the effect of random genetic drift will be considered at a later stage. The selection is of the optimum type so that we can examine the change in variance under two situations: (a) a population that evolves from monomorphism (at an optimum phenotype), and (b) when an equilibrium population shifts to a new environment where the optimum phenotype is shifted by a few units of the phenotypic scale. The transient behaviour of the approach to equilibrium is studied in terms of changes in means as well as variance. The changes in the interpopulational variance is examined when an equilibrium population splits into two, where one of them moves to a new optimum environment.

\section{THE MODEL}

We consider modelling for those quantitative characters which are controlled by $n$ loci where $n$ is usually small say 5 to 6 . We assume that at each locus there is an infinite number of possible allelic states. For a given locus, let $\boldsymbol{A}_{i}$ represent an allele occupying state $i$ (any integer number from $-\infty$ to $\infty$ ) and having an allelic effect of $i a$. We assume that all allelic effects are additive with no dominance and no epistasis and that once $A_{i}$ mutates, it changes to allelic state $(i+r) a$ with probability,

$$
\begin{aligned}
& a_{r}=a_{\cdot r}=\left(\begin{array}{c}
2 m \\
m-r
\end{array}\right)\left(\frac{1}{2}\right)^{2 m} \text { for } 0 \leq r \leq m \\
& a_{r}=0 \text { otherwise }
\end{aligned}
$$

where $m$ is the possible number of mutational changes. (1) is a shifted binomial distribution with mean and variance of the distribution at 0 and $m / 2$ respectively. If $v$ denotes the mutation rate, the absolute probability of such a mutation would be $v a_{r}$. Thus, an allele that mutates but has the same allelic affect as that of the original allele would do so with probability $v a_{0}$, so that in the conventional definition, the real mutation rate $v^{\prime}$ would be given by $v^{\prime}=\left(1-a_{0}\right) v$. The increment of the variance of allelic effect by mutation is then $v m a^{2} / 2$ per generation as shown in Clayton and Robertson (1955). This being true for haploids, the corresponding value for diploids would be $\sigma_{m}^{2}=$ $v m a^{2}$.

The selection operates on the total phenotypic value $y$. With no genotype-environment interaction and taking the case of a single locus with multiple 
alleles, the distribution of $y$, among individuals of type $A_{i} A_{j}$ with genotypic value $a(i+j)$ follows a normal distribution with mean $a(i+j)$ and the environmental variance $\sigma_{e}^{2}$. The fitness function for the character value $y$ is assumed to be Gaussian type as

$$
w(y)=w_{\max } \exp \left\{-\frac{\left(y-y_{\mathrm{opt}}\right)^{2}}{2 \sigma_{w}^{2}}\right\}
$$

where the quantitative character is assumed to be optimum for fitness at $y=y_{\text {opt }}$ with maximum fitness of $w_{\max }$ and $\sigma_{w}$ is the width of the function indicating the rate at which fitness declines with deviation of $y$ from the optimum. Taking $w_{\max }=1$, and as in Lande (1975) the mean fitness of the individuals with genotype $\boldsymbol{A}_{i} \boldsymbol{A}_{j}$ would be

$$
w_{i j} \alpha \exp \left[-s\left\{a(i+j)-y_{\mathrm{opt}}\right\}^{2}\right]
$$

where $s=1 / 2\left(\sigma_{w}^{2}+\sigma_{e}^{2}\right)$ indicates the strength of the selection at the group level. A large $\sigma_{w}$ means small $s$ and therefore would indicate weak selection of optimum type whereas a small $\sigma_{w}$ means large $s$ resulting in strong selection of optimum type. Since the contribution of the given locus to the phenotypic variance is taken as small, the heritability of the character would be rather low and deviations from normality in the distribution of genotypic effects could be important as we investigate below.

\section{THEORY}

\section{One locus: optimum phenotype at origin}

If $x_{i}(t)$ denotes the frequency of allele $A_{i}$ in generation $t$ with allelic effect $a i$, an individual of genotype $A_{i} A_{j}$ will have a mean fitness $w_{i j}=$ $\exp \left[-s a^{2}(i+j)^{2}\right]$ with the optimal value of $y$ as zero and thus, the change in gene frequency of $A_{i}$ from generation $(t-1)$ to $t$ is given by

$$
\begin{aligned}
& \bar{w}_{A}(t-1) x_{i}(t) \\
&=\left(1-v+v a_{0}\right) \sum_{j} x_{i}(t-1) x_{j}(t-1) \\
& \times \exp \left[-s a^{2}(i+j)^{2}\right] \\
&+v \sum_{r=1}^{m} a_{r} \sum_{j} x_{j}(t-1)\left[x_{i+r}(t-1)\right. \\
& \times \exp \left\{-s a^{2}(i+j+r)^{2}\right\} \\
&\left.+x_{i-r}(t-1) \exp \left\{-s a^{2}(i+j-r)^{2}\right\}\right]
\end{aligned}
$$

where $\bar{w}_{A}(t-1)$ is the mean fitness of individuals at the locus in the $(t-1)$ th generation so adjusted as to make $\sum_{j} x_{j}(t-1)=1$.
In general, this recurrence relationship does not yield any explicit solution. However, for $m=1$ it is possible to derive the equilibrium allele frequency profile by neglecting powers of $v$ and $s$ as well as their products. The mean fitness $\bar{w}_{A}(t)$ is then approximated as

$$
\begin{aligned}
\bar{w}_{A}(t) & \cong 1-s a^{2} \sum_{i} \sum_{j} x_{i}(t) x_{j}(t)(i+j)^{2} \\
& =1-s \sigma_{g_{A}}^{2}(t)
\end{aligned}
$$

where $\sigma_{g_{A}}^{2}(t)$ is the total genotypic variance contributed by this locus at time $t$.

Since the optimum genotype is at the origin, we have initially $x_{i}(0)=x_{-i}(0)$. Then for all $i$, $x_{i}(t)=x_{-i}(t)$ at each generation, this property being invariant to the transformation (4). From the symmetry of the model, we expect the equilibrium to be globally stable. As shown in Appendix $A$ at equilibrium, the frequent allele frequencies are obtained as

$$
\begin{aligned}
& \hat{x}_{1}=\hat{x}_{0}(1-S G) \\
& \hat{x}_{2}=\hat{x}_{0}[1-2 S G+2 S(1-G)(1-S G)]
\end{aligned}
$$

and

$$
\begin{aligned}
\hat{x}_{3} & =\hat{x}_{0}[1-3 S G+6 S(1-G)(1-S G) \\
& +2 S\{3-S G(6-G) \\
& +2 S(1-G)(4-G)(1-S G)\}]
\end{aligned}
$$

where $S=s a^{2} / v$ and $G=\frac{1}{2} \sum_{i} \sum_{j} \hat{x}_{i} \hat{x}_{j}(i+j)^{2}=$ $\sigma_{g_{A}}^{2} / 2 a^{2}$, with $\hat{x}_{i}=\hat{x}_{-1}$ for all $i=1,2, \ldots$ This analytical approximation for equilibrium profile is similar to that of Gillespie (1984) though only vaguely since mutation is not incorporated in that study.

In the general case of $m$-step mutational changes, the moments of the allelic effects as well as those of genotypic effects can be obtained analytically under optimum selection and the same assumptions for $v$ and $s$ as those used for $m=1$. Denoting the $k$ th moment of the distribution of allelic effects at a locus in the $t$-th generation by $M_{k}(t)=\sum_{i=-\infty}^{\infty} a^{k} i^{k} x_{i}(t)$, the recurrence relationship for the even order moments is approximately given by

$$
\begin{aligned}
M_{2 k}(t) \cong & {\left[1-v+v\left(\begin{array}{c}
2 m \\
m
\end{array}\right)\left(\frac{1}{2}\right)^{2 m}\right] M_{2 k}(t-1) } \\
& +s\left[M_{2}(t-1) M_{2 k}(t-1)-M_{2 k+2}(t-1)\right] \\
& +2 v \sum_{l=0}^{k}\left(\begin{array}{c}
2 k \\
2 l
\end{array}\right) M_{2 l}(t-1) \\
& \times \sum_{r=1}^{m}\left(\begin{array}{c}
2 m \\
m-r
\end{array}\right)\left(\frac{1}{2}\right)^{2 m}(a r)^{2 k-2 l}
\end{aligned}
$$


so that the change of variance of allelic effects at generation $t, \Delta M_{2}(t)$, is given by

$$
\Delta M_{2}(t)=\frac{m v a^{2}}{2}+s\left[M_{2}^{2}(t)-M_{4}(t)\right] .
$$

At equilibrium, therefore, the fourth moment and the variance of allelic effects are related by

$$
\hat{M}_{4}=\hat{M}_{2}^{2}+\frac{m v a^{2}}{2 s}
$$

Now, if $\mu_{r}(t)$ denotes the $r$-th order moment of the genotype effects at the locus (i.e., $\mu_{r}(t)=$ $\left.\sum_{i} \sum_{j} a^{r}(i+j)^{r} x_{i}(t) x_{j}(t)\right)$, the variance and the fourth moments of the genotypic effect at a locus are related with those of allelic effects by the relation

$$
\mu_{2}(t)=2 M_{2}(t) \text { and } \mu_{4}(t)=2 M_{4}(t)+6 M_{2}^{2}(t) .
$$

under the assumption that $v$ and $s$ are so small that their powers and products could be neglected.

From eq. (8) we thus obtain the change of variance of genotypic values at a locus in generation $t, \Delta \mu_{2}(t)$, approximately as

$$
\Delta \mu_{2}(t) \cong m v a^{2}-s\left[\mu_{4}(t)-2 \mu_{2}^{2}(t)\right] .
$$

When the equilibrium distribution of genotypic values is taken as normal (i.e., $\hat{\mu}_{4}=3 \hat{\mu}_{2}^{2}$ ), the genotypic variance at a locus in the equilibrium population is given by $\hat{\sigma}_{g_{1}}^{2}=\hat{\mu}_{2}=\sqrt{m v a^{2} / s}=$ $\sqrt{\sigma_{m}^{2} / s}$. This result is identical to that of Kimura (1965) even though his model assumes continuous time parameter and a continuous distribution of allelic effects.

We, therefore, obtain the important result of non-normal equilibrium distribution under the discrete allelic effect model. The departure from normality can be determined from the value of $\hat{\beta}_{2}=$ $\hat{\mu}_{4} / \hat{\mu}_{2}^{2}$ at equilibrium. From eq. (11) we obtain

$$
\hat{\beta}_{2}=2+\sigma_{m}^{2} / s \hat{\sigma}_{g_{A}}^{4},
$$

and hence the departure from normality as measured by $\gamma=\hat{\beta}_{2}-3$ is given by

$$
\begin{aligned}
\gamma & =\left(\sigma_{m}^{2}-s \hat{\sigma}_{g_{A}}^{4}\right) / s \hat{\sigma}_{g_{A}}^{4} \\
& =\left(\frac{m v \alpha^{2}}{4 I h_{A}^{4}}-1\right)
\end{aligned}
$$

where $\alpha$, the proportionate effect of the locus, as defined by Falconer $(1960)$, is $\left(2 a / \sigma_{p}\right)$ and $I$ is the intensity of natural selection as given in Haldane (1954).
Thus, in general with a step-wise mutation model under optimising selection, the equilibrium distribution of genotypic values is not normal. Fleming (1979) also obtained with continuous allelic-state model that genotypic values, at equilibrium, are not normally distributed. This was also confirmed by Turelli (1984, 1985).

One locus: optimum d s.d. units away from the origin

Let us now consider the case where the population which is at equilibrium initially with mean phenotype at the origin and variance $\sigma_{p}^{2}$ now shifts to a new environment where the optimum phenotype is $d \sigma_{p}$ units away from the mean. Obviously, under the effect of mutation and selection the equilibrium status of genotypic distribution will be immediately disturbed and gradually the distribution will shift towards the new optimum. To analyze the nature and rate of change of variability we must again consider the recurrence relationship of gene frequency changes. In this new environment the fitness, $w_{i j}$, of an individual of genotype $A_{i} A_{j}$ is given by $w_{i j}=$ $\exp \left[-s\left\{a(i+j)-d \sigma_{p}\right\}^{2}\right]$.

The recurrence relationship of the gene frequencies in two successive generations with $\mathrm{m}$ step mutation model is then given by

$$
\begin{aligned}
\bar{w}(t-1) & x_{i}(t) \\
= & {\left[1-v\left\{1-\left(\begin{array}{c}
2 m \\
m
\end{array}\right)\left(\frac{1}{2}\right)^{2 m}\right\}\right] } \\
& \times \sum_{j} x_{i}(t-1) x_{j}(t-1) \\
& \times \exp \left\{-s a^{2}\left(i+j-\frac{d}{a} \sigma_{p}\right)^{2}\right\} \\
& +v \sum_{j} \sum_{r=1}^{m} x_{j}(t-1)\left(\begin{array}{c}
2 m \\
m-r
\end{array}\right)\left(\frac{1}{2}\right)^{2 m} \\
& \times\left[x_{i+r}(t-1)\right. \\
& \times \exp \left\{-s a^{2}\left(i+j+r-\frac{d}{a} \sigma_{p}\right)^{2}\right\} \\
& +x_{i-r}(t-1) \\
& \left.\times \exp \left\{-s a^{2}\left(i+j-r-\frac{d}{a} \sigma_{p}\right)^{2}\right\}\right]
\end{aligned}
$$

As before, we assume that $v$ and $s$ are so small that their powers as well as products could be 
neglected. The mean fitness $\bar{w}(t)$ is then approximated as

$$
\bar{w}(t) \cong 1-s\left[\sigma_{g_{A}}^{2}(t)+\left\{\mu_{1}^{\prime}(t)-d \sigma_{p}\right\}^{2}\right]
$$

where $\mu_{1}^{\prime}(t)$ is the average genotypic value in the population at generation $t$.

Using this expression of $\bar{w}(t)$ in eq. (13) and multiplying both sides by $a i$, a summation over all $i$ yields the recurrence relationship connecting the mean genotypic values in two successive generations which is given by

$$
\begin{aligned}
\mu_{1}^{\prime}(t)= & \mu_{1}^{\prime}(t-1)-s\left[\mu_{3}(t-1)+2 \mu_{2}(t-1)\right. \\
& \left.\times\left\{\mu_{1}^{\prime}(t-1)-d \sigma_{p}\right\}\right]
\end{aligned}
$$

where $\mu_{1}^{\prime}(t)$ is the $r$-th order moment (about origin) of the genotypic values in the $t$ th generation. Clearly, at equilibrium we thus have $\mu_{1}^{\prime}=$ $d \sigma_{p}$, the optimum genotypic value and $\mu_{3}=0$, the equilibrium distribution being symmetric.

The change of genotypic variance can be similarly obtained by multiplying both sides of eq. (13) by $a^{2} i^{2}$ and summing over all $i$. After some algebraic simplifications we obtain, approximately.

$$
\begin{aligned}
\Delta \sigma_{g_{A}}^{2}(t)= & m v a^{2}+s\left[2 \sigma_{g_{A}}^{4}(t)\right. \\
& \left.+\mu_{3}(t)\left\{\left(d \sigma_{p}\right)-2 \mu_{1}^{\prime}(t)\right\}-\mu_{4}(t)\right]
\end{aligned}
$$

where $\mu_{3}(t), \mu_{4}(t)$ represent the third and fourth moments (about mean) of genotypic values at the $t$-th generation. At equilibrium, since $\hat{\mu}_{3}$ is zero (as the distribution is symmetric), we again have the steady state genotypic variance approximately given by

$$
\hat{\sigma}_{g_{A}}^{2} \cong \sqrt{\frac{1}{2}\left\{\mu_{4}-\frac{m v a^{2}}{s}\right\}}
$$

which is identical to the expression as obtained from eq. (11). Thus, even if the optimum is shifted by a certain s.d. away from the original mean, as long as the intensity of selection $(s)$ remains the same, the genotypic variance eventually returns to its original value although the genotypic distribution becomes now centred around the new optimum genotypic value.

At the transitory stage it is difficult to assert analytically from eq. (16) how the variance is altered. However, as we shall see in our numerical computations, at a transitory stage the genotypic variance first increases and eventually returns to its original equilibrium value.

\section{Two loci: optimum selection model}

We now consider two linked loci $A$ and $B$ at each of which an infinite number of possible allelic types can exist. Under the assumption that allelic effects over loci are additive without any epistatic interaction, let the contribution of the gametic effect of the gamete $A_{i} B_{j}$ be represented by $a(i+j)$. Fig. 1

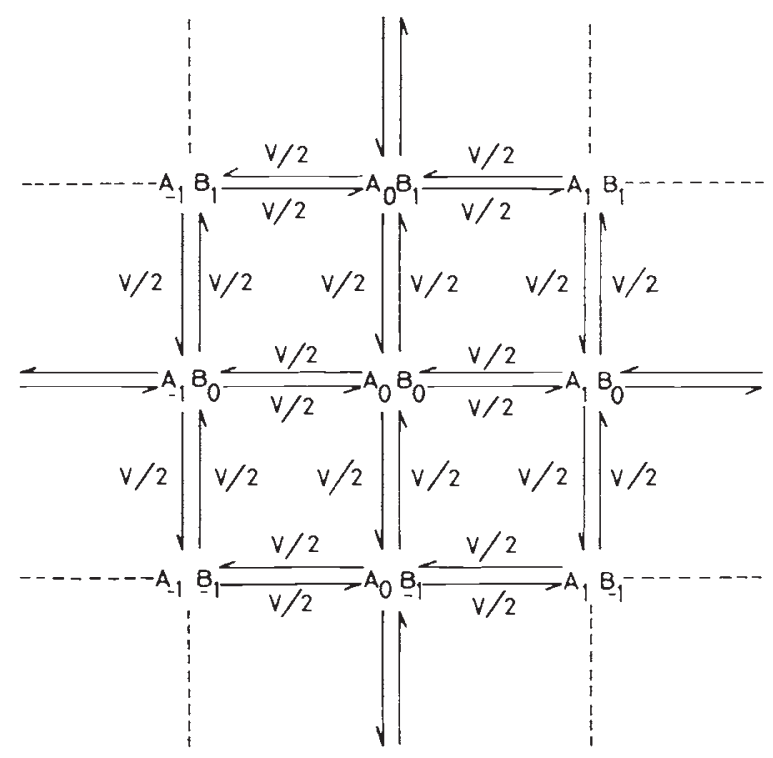

Figure 1 Two-locus allelic-state model used in this paper. In this model the gamete $A_{i} B_{j}$ mutates to $A_{i} B_{j \pm 1}$ or $A_{i \pm 1} B_{j}$ with probability $v / 2$. The alleles $A_{i}$ and $B_{j}$ have phenotypic effects $a i$ and $a j$, respectively.

represents the mutational scheme which is essentially a two-dimensional extension of Chakraborty and Nei's (1982) scheme with $m=1$ in both dimensions ( $A$ and $B$ loci). Under this scheme the gamete type $A_{i} B_{j}$ can mutate to $A_{i+1} B_{j}, A_{i-1} B_{j}$, $A_{i} B_{j+1}$ or $A_{i} B_{j-1}$ each with probability $v / 2$ whereas it does not mutate and therefore has the same phenotypic effect as that of the original gamete with probability $(1-2 v)$. Effectively we assume that mutations of type $A_{i} B_{j}$ to $A_{i \pm 1} B_{j \pm 1}$ are of negligible order. The mean fitness of individuals with genotype $A_{i} A_{j} B_{k} B_{l}$ is assumed to be $w_{i j k l}=$ $\exp \left[-s a^{2}(i+j+k+l)^{2}\right]$ with optimal value of the characters at zero (optimal genotype being $\left.A_{0} A_{0} B_{0} B_{0}\right)$ with relative fitness of unity. Let the recombination value between the two loci be $r$. Assuming that selection, recombination and mutation events occur as in the stated order, the recurrence relationship between the gametic frequencies 
in two successive generations is given by

$$
\begin{aligned}
\bar{w}_{A B}(t- & 1) X_{i j}(t) \\
= & (1-2 v) \sum \sum e^{-s a^{2} z^{2}} \\
& \times\left[(1-r) X_{i j}(t-1) X_{k l}(t-1)\right. \\
& \left.+r X_{i}(t-1) X_{k j}(t-1)\right] \\
& +\frac{v}{2} \sum_{k} \sum_{l}\left[e ^ { - s a ^ { 2 } ( z + 1 ) ^ { 2 } } \left\{(1-r) X_{k l}(t-1)\right.\right. \\
& \times\left(X_{i+1, j}(t-1)+X_{i, j+1}(t-1)\right) \\
& +r\left(X_{i+1, l}(t-1) X_{k j}(t-1)\right. \\
& \left.\left.+X_{i, l}(t-1) X_{k, j+1}(t-1)\right)\right\} \\
& +e^{-s a^{2}(z-1)^{2}}\left\{(1-r) X_{k l}(t-1)\right. \\
& \times\left(X_{i-1, j}(t-1)+X_{i, j-1}(t-1)\right) \\
& +r\left(X_{i-1, l}(t-1) X_{k j}(t-1)\right. \\
& \left.\left.\left.+X_{i, l}(t-1) X_{k, j-1}(t-1)\right)\right\}\right]
\end{aligned}
$$

where $\bar{w}_{A B}(t-1)$ is the mean fitness of the population at generation $(t-1)$, and $z=i+j+k+l$. As before, neglecting powers of $s, v$ and $s v$, we have

$$
\begin{aligned}
\bar{w}_{A B}(t-1) X_{i j}(t) \\
\simeq \sum_{k} \sum_{l}\left(1-2 v-s a^{2} z^{2}\right) \\
\quad \times\left[(1-r) X_{i j}(t-1) X_{k l}(t-1)\right. \\
\left.\quad+r X_{i l}(t-1) X_{k j}(t-1)\right] \\
+\frac{v}{2}\left[( 1 - r ) \left\{X_{i+1, j}(t-1)+X_{i-1, j}(t-1)\right.\right. \\
\left.+X_{i, j+1}(t-1)+X_{i, j-1}(t-1)\right\} \\
\quad+r\left\{x_{i+1}(t-1) y_{j}(t-1)\right. \\
+x_{i}(t-1) y_{j+1}(t-1)+x_{i-1}(t-1) y_{j}(t-1) \\
\left.\left.\quad+x_{i}(t-1) y_{j-1}(t-1)\right\}\right]
\end{aligned}
$$

where $\quad x_{i}(t-1)=\sum_{j} X_{i j}(t-1) \quad$ and $\quad y_{j}(t-1)=$ $\sum_{i} X_{i j}(t-1)$ are the gene frequencies of alleles $A_{i}$ and $B_{j}$ at the $A$ and $B$ locus, respectively in $(t-1)$ th generation.

It is easy to see that under the above approximation the mean fitness $\bar{w}_{A B}(t-1)$ at generation $t$ is given by

$$
\begin{aligned}
\bar{w}_{A B}(t) & \cong 1-s a^{2} \sum_{i} \sum_{j} \sum_{k} \sum_{l}(i+j+k+l)^{2} X_{i j}(t) X_{k l}(t) \\
& =1-s \sigma_{g_{A B}}^{2}(t)
\end{aligned}
$$

where $\sigma_{g_{A B}}^{2}(t)$ is the genotypic variance at the $t$-th generation.

In general, analytic expressions for the recurrence relationship for the moments of the genotypic distribution are tedious to obtain. However, it is easy to see that if the population is at linkage equilibrium (i.e., $D_{i j}(t)=X_{i j}(t)-$ $x_{i}(t) y_{j}(t)=0$ for all $i$ and $j$ ), eq. (19) will yield the corresponding one locus recurrence equations.

Once the gametic frequencies in a particular generation are obtained from eq. (19) by iterative evaluations, we can obtain the distribution of genotypic values and its various statistics (e.g., mean, variance, skewness and kurtosis) by forming genotypes by random union of gametes. The disequilibrium of allele frequencies at $A$ and $B$ loci are studied by a statistic of the form

$$
D^{2}(t)=\frac{\sum_{i} \sum_{j} D_{i j}^{2}(t)}{H_{A}(t) H_{B}(t)}
$$

where $H_{A}(t)$ and $H_{B}(t)$ are the underlying heterozygosities at the $A$ and $B$ loci, respectively (i.e., $H_{A}(t)=1-\sum_{i} x_{i}^{2}(t)$ and $\left.H_{B}(t)=1-\sum_{j} y_{j}^{2}(t)\right)$.

\section{RESULTS}

It is apparent from the theoretical development given in the previous section that a quantitative study of genetic differentiation for metric traits between populations of species cannot be made analytically if optimal selection with stepwise mutation in an infinite population is envisaged. A computer was therefore used to compute numerically the various quantities of interest by resorting to exact recurrence relations for single as well as two-loci models already discussed in the previous sections. The mean and variability of the character both within as well as between populations were studied in the transient stage and at equilibrium. To start with, the initial population was considered as monomorphic at optimum i.e., genetically homogeneous with only one type of individuals and the behaviour of within population variance studied over time. After reaching the equilibrium, the optimum was shifted to a few units on the right and the transient behaviour of the mean and variability (within as well as between) was studied. In so far as within population variability with optimum at origin is concerned, detailed discussions of equilibria have since appeared in Nagylaki (1984) and Turelli (1984, 1985). However, when the optimum is shifted, on reach- 
ing equilibrium, the transient behaviour of the variance and skewness does not seem to have appeared in print. The discussion of such behaviour by Gillespie (1984) pertains to a different situation as it does not involve mutation.

\section{Within population variability}

Since the mean is at the optimum and the optimum is set at zero, the population mean remains at zero unless there is a shift of the optimum genotype. However, the variance increases slowly from zero and attains, at equilibrium, a value determined solely by $(v / s)$ as already obtained algebraically from relation (11). This behaviour of within population variance at equilibrium for varying values of $s$ between 0.004 to 0.040 for $m=1$ and $v=$ 0.0005 is presented in table 1 . The results are found to be comparable to those given in Turelli (1984).

Table 1 Within population variance at equilibrium when initial population is genetically homogeneous with only one type of individuals which is optimum phenotype for some selected values of selection coefficients $(s)$. The mutation rate, $v$, is taken as 0.0005 and $m=1$

\begin{tabular}{ll}
\hline$s$ & $\hat{\sigma}_{g_{A}}^{2}$ \\
\hline 0.004 & $1.17 \times 10^{-1}$ \\
0.008 & $6.03 \times 10^{-2}$ \\
0.010 & $4.85 \times 10^{-2}$ \\
0.020 & $2.44 \times 10^{-2}$ \\
0.040 & $1.22 \times 10^{-2}$ \\
\hline
\end{tabular}

For more intense selection, the variances at equilibrium are lower as otherwise expected. Mutation creates variability while selection eliminates it so that for intense selection, its role is dominant. Also, the approach to equilibrium is found to be quicker for more intense selection as revealed by results of computer simulations which are not presented here.

\section{Between population variability}

When we shift the optimum to four or six standard deviation units away from the mean on the right and study the transient behaviour of the process as it approaches the same equilibrium, we notice some interesting results. It may be noted that such drastic changes in the optimum are consistent with the type of quantitative traits aimed at in this study such as skin pigmentation. It is believed that a small group of Caucasian race with fair skin moved out of Central Asia around 3000 years ago and settled in southern parts of America with plenty of sunlight. Their skin pigmentation must have changed due to a drastic shift in the optimum phenotype for pigmentation. Size of tooth in prehistoric man is another such example where optimum phenotype might have shifted drastically due to changed environmental conditions. It is therefore biologically relevant to assume that a population which reached a steady state by mutation-selection balance now splits into two, one of which moves to a new environment which shifts the optimal genotype 4 to 6 standard deviations away from the origin which was the original genotypic value. As soon as this occurs, the corresponding daughter population starts differentiating from its sister population effectively by favouring certain classes of mutant alleles and discarding others which were originally favoured. During this process of turn over of genes, the statistical properties of the allelic distribution undergo characteristic changes, depending upon the magnitudes of $v$, $s$, and $m$. In table 2 we present these results in terms of mean, variance, skewness, and kurtosis of genotypic values in different generations for a quantitative character under centripetal selection when the new optimum is at six standard deviations away from the original optimum. We take $v=$ $0.001, s=2 v$, and $m=5$. A somewhat higher value of mutation rate than warranted by empirical estimates as given in Turelli (1984) was taken to speed

Table 2 Mean $\left(\bar{X}_{t}\right)$, variance $\left(V_{t}\right)$, skewness $\left(\gamma_{1 t}\right)$ and kurtosis $\left(\gamma_{2 t}\right)$ of genotypic values in different generations for a quantitative trait under centripetal selection. $(v=0.001, s=2 v$ and $m=5$ ) with new optimum 6 standard deviations away from original optimum

\begin{tabular}{llllrrr}
\hline & \multicolumn{7}{c}{$t$} \\
\cline { 2 - 7 } & 0 & 50 & 100 & 500 & 1000 & $\infty$ \\
\hline Mean $\left(\bar{X}_{t}\right)$ & 0 & 0.378 & 0.930 & 3.443 & 3.811 & 4.000 \\
Variance $\left(V_{t}\right)$ & 0.456 & 0.728 & 1.296 & 0.815 & 0.618 & 0.456 \\
Skewness $\left(\gamma_{1 t}\right)$ & 0 & 0.997 & 0.684 & 0.119 & -0.001 & \multicolumn{1}{l}{0} \\
Kurtosis $\left(\gamma_{2 t}\right)$ & 2.741 & 1.886 & 0.433 & 0.764 & 1.563 & 2.741 \\
\hline
\end{tabular}

N.B. Kurtosis $\left(\gamma_{2 t}\right)$ is measured by $\gamma_{2 t}=\left(\mu_{4 t} / V_{t}^{2}\right)-3$. 
up the simulations. The selection intensity was taken as twice the mutation rate. However, the qualitative results seem to be true with lower values of mutation rates coupled with higher values of selection intensities. Since selection intensity is taken as greater than mutation rate, the results presented tend to have biological relevance in the context of arguments on the relative magnitudes of these parameters given in Turelli (1984).

The mean increases from zero to four at equilibrium but the increase is more rapid for a higher value of $m$. The variance on the other hand increases, attains a transitory maximum and decreases back to the original steady state value $\left(V_{0}=V_{\infty}\right)$ which is related to the fourth moment of the genotypic values as given by (17) where $V_{\infty}$ is $\hat{\sigma}_{g_{A}}^{2}$. When $s$ is very large compared to $v$, more than one transitory maxima are produced. In fig. 2 , we present such a behaviour of intra-populational variance for $s=0 \cdot 002,0.004$ and 0.008 when $v=0.001, m=1$ and when the new optimum is at six standard deviations away from the original optimum at the origin.

For higher values of $m$, the variance attains a considerably higher peak as well as somewhat earlier than when $m=1$. The most interesting feature is regarding the skewness of the distribution of genotypic values. Initially, this distribution is symmetrical but as we advance in time, its symmetry is disturbed. It gets skewed initially and then slowly the skewness decreases, changes sign and finally the distribution becomes again symmetrical at equilibrium. The kurtosis of the distribution also behaves in a similar fashion. Starting from a value very near to three initially, it declines to a value less than half but increases thereafter and restores the initial value at the equilibrium. Compared to $m=1$, the distribution for $m=5$ becomes more leptokurtic. A typical allelic distribution corresponding to $v=0 \cdot 001, s=2 v$ and $m=5$, depicting these features, is shown in fig. 3.

Of special importance in these studies is the genetic differentiation between populations built up over a period of time when a population which reached a steady state by mutation-selection balance splits into two in one of which the same optimum holds but in the other it has shifted a certain distance away from the mean. As soon as this occurs the corresponding daughter population starts differentiating from its sister population effectively by favouring certain classes of mutant alleles and discarding others which were originally favoured. We have already discussed in the previous section how intra-poyulation variance $\left(V_{t}\right)$ behaves under such circumstances. Since the mean genotypic values of the two populations diverge, a between-population variance $\left(B_{t}\right)$ is built up over time in a characteristic manner. This variance can be measured as one-fourth of the square of the difference of mean genotypic values of the two populations genetically isolated for $t$ generations. In Chakraborty and Nei (1982) to obtain this vari-

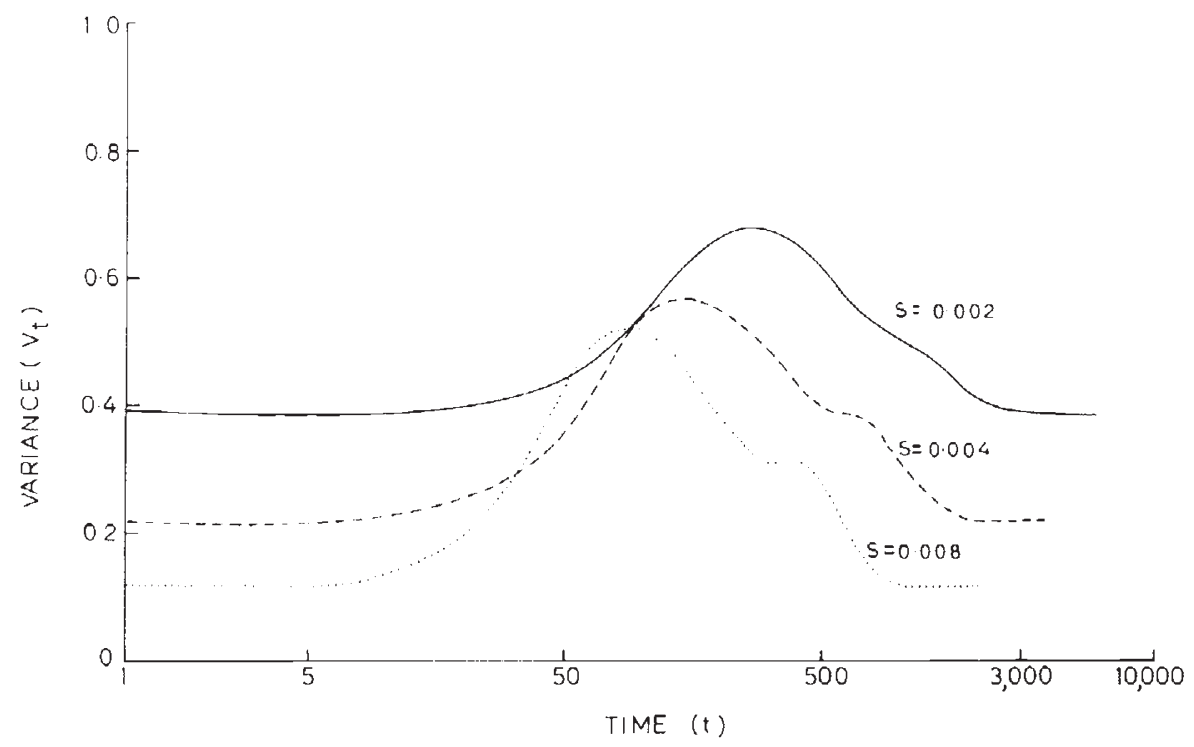

Figure 2 Intra-population variance $\left(V_{t}\right)$ under the joint effects of mutation and centripetal selection as a function of time $t$, with $m=1, v=0.001, s=0.002,0.004$ and 0.008 when the new optimum is at 6 standard deviations away from the original optimum at the origin. Initial population is at steady-state. 


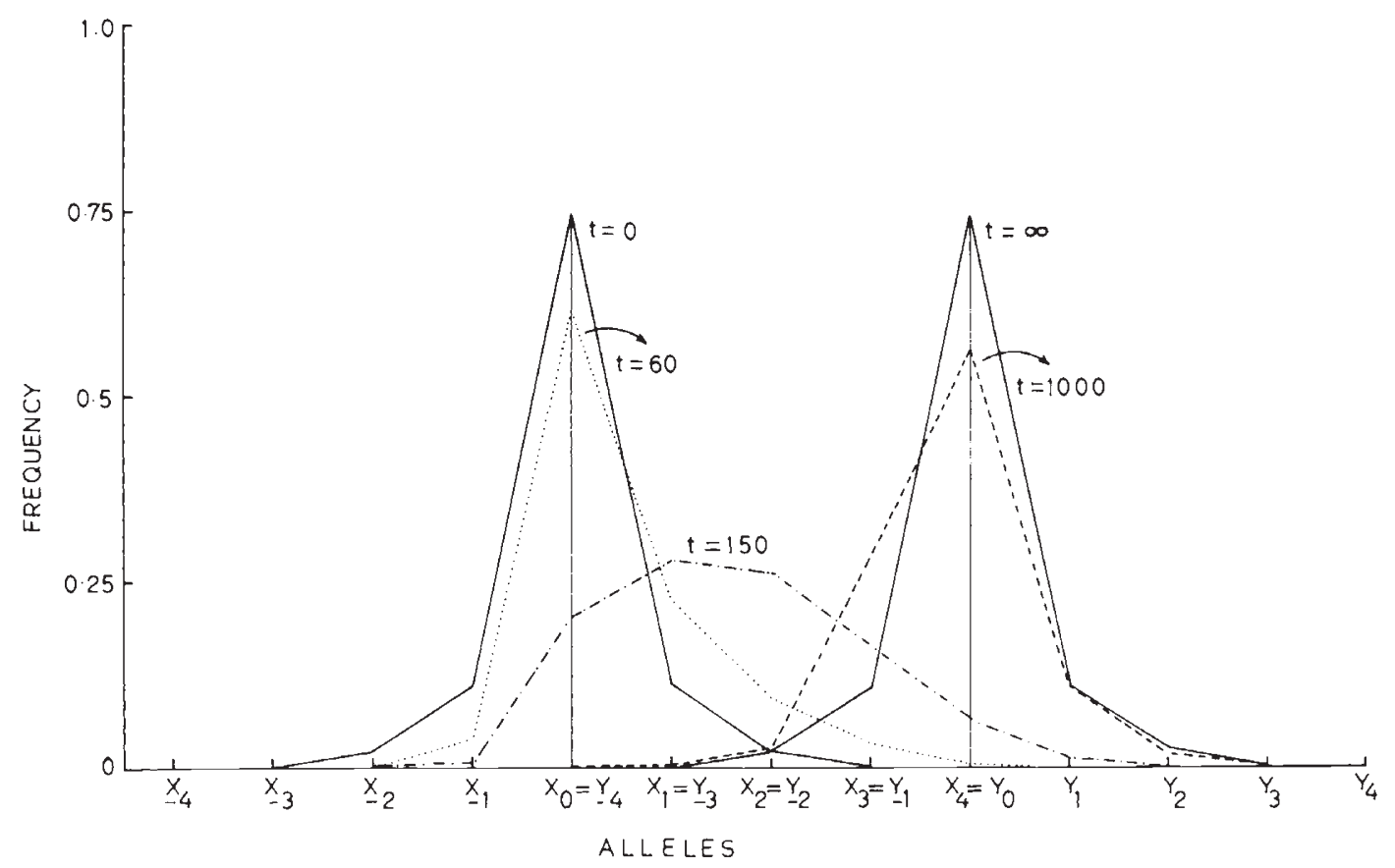

Figure 3 Allele frequency distribution under joint effects of mutation and centripetal selection at different generations $(t=0$, 60 , 150,1000 , and $\infty$ ) with $v=0 \cdot 001, s=20$ and $m=5$ when the new optimum is 6 standard deviations away from the original optimum at the origin. Initial population is at steady-state.

ance, two randomly chosen alleles one from each of the two populations are considered and variance $\left(D_{t}\right)$ of the difference between these two alleles is expressed as

$$
D_{t}=V_{t}+B_{t} /(2 n)
$$

where the character is assumed to be controlled by $n$ loci at each of which the allelic effects are identical and proportional to their states.

With no selection and for a finite population of size $N$, they obtain expressions for $B_{t}$ and $V_{t}$ as

$$
\begin{aligned}
& B_{t}=2 n m v a^{2} t+\left(V_{0}-2 N n m v a^{2}\right)\left(1-e^{-t / 2 N}\right) \\
& V_{t}=2 N n m v a^{2}+\left(V_{0}-2 N n m v a^{2}\right) e^{-t / 2 N}
\end{aligned}
$$

where $V_{0}$ represents the genetic variance of the parental population at the time when split occurs. When the initial population is at mutation-drift balance,

$$
V_{0}=V_{\infty}=2 N n m v a^{2}
$$

and therefore $B_{t}$ increases linearly with time of divergence from the very start of genetic differentiation but $V_{t}$ remains constant. Hence the variance ratio $\left(B_{t} / V_{t}\right)$ is perfectly correlated with the time of divergence $(t)$. However, when centripetal selection in an infinitely large population, as in the present study, is considered, $B_{t}$ monotonically increases in a non-linear fashion reaching a steady state value as the population in the new environment approaches its steady-state distribution. $V_{t}$, on the other hand, first starts increasing over the equilibrium value $V_{\infty}$ and after crossing one or more transitory maxima again returns to the steady-state value $V_{\infty}$. The behaviour of $\left(B_{t} / V_{t}\right)$ as a function of time of divergence, is consequently non-linear in fashion. Thus a comparison of this ratio in the two situations, with or without selection, may help in understanding the role of adaptive changes under which the character changes over time. However, the non-linear relationship of the time of divergence and this ratio cannot be taken as a definitive proof of the adaptive nature of the trait under consideration. To illustrate the qualitative nature of the changes in $V_{t}, B_{t}$ and $\left(B_{t} / V_{t}\right)$, under joint effects of mutation and centripetal selection, fig. 4 presents the numerical results for $v=0.001, s=2 v$ and $m=5$ when the new optimum is taken to be approximately 6 standard deviations away from the optimum in the other population.

It is seen from this figure that the rate of approach to equilibrium variance within populations $\left(V_{\infty}=0.456\right)$ is faster as compared to that of 


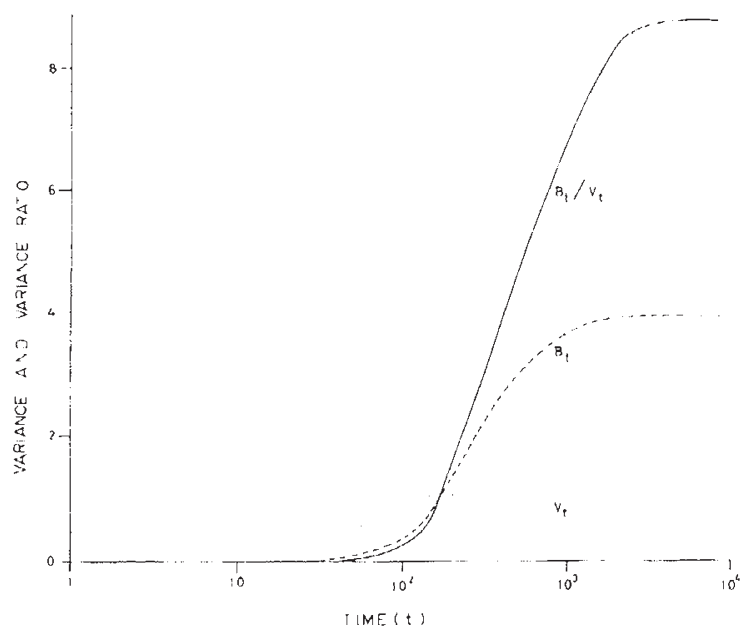

Figure 4 Intra- $\left(V_{t}\right)$ and inter-populational $\left(B_{t}\right)$ variance components under the joint effects of mutation and centripetal selection as a function of time of divergence of two populations one of which has an optimum genotype six standard deviations away from the other (which remains at a steady state by mutation-selection balance). Time $(t)$ is measured in units of generation. Initial population is at steady state. The parameter values are $v=0.001, s=2 v$, and $m=5$.

the mean genotypic value $\left(\bar{X}_{\infty}=4 \cdot 0\right)$ since the between-population variance $\left(B_{t}\right)$ attains its equilibrium value $\left(B_{\infty}=4.0\right)$ at a later time than $V_{t}$, the within population variance. Unlike the nonadaptive trait case discussed in Chakraborty and Nei (1982), under centripetal selection and in an infinite population, the variance ratio $\left(B_{t} / V_{t}\right)$ reaches a steady state value $\left(B_{\infty} / V_{\infty}=8 \cdot 80\right)$ since the process of genetic differentiation stops once the diverging population reaches its steady-state genotypic distribution around its new optimum genotypic value which is 6 standard deviations away from the original optimum at origin. We thus see that with selection the ratio $\left(B_{t} / V_{t}\right)$ asymptotes and does not therefore increase linearly with time as found in Chakraborty and Nei (1982) where selection is ignored and population size is finite.

The results presented above relate to selection at the genotypic level when single locus is considered. However, it was also possible to obtain similar results for the two-loci case when gametic selection is resorted to. These results pertaining to within population variability are presented in table 3 , where the initial population is taken as genetically homogeneous with only one type of optimal genotype and recombination fractions take three different values between complete linkage and no linkage, viz. $0 \cdot 0,0 \cdot 25$, and $0 \cdot 50$. It is apparent that the effect of linkage is not very pronounced in the initial stages but at equilibrium it is found to reduce
Table 3 Genetic variance $\left(V_{t}\right)$ within a population of a character controlled by two loci under the joint effect of mutation and centripetal selection as a function of time ( $t$, in generations) and recombination fraction $(r)$. (Population size infinite, $v=$ $0.001, s=5 v, m=1$; initial population genetically homogeneous with only one type of optimal genotype)

\begin{tabular}{rlll}
\hline & \multicolumn{2}{c}{$r$} \\
\cline { 2 - 4 }$t$ & 0.0 & 0.25 & 0.50 \\
\hline 10 & 0.039 & 0.039 & 0.039 \\
50 & 0.175 & 0.175 & 0.176 \\
100 & 0.303 & 0.308 & 0.309 \\
500 & 0.618 & 0.672 & 0.674 \\
1000 & 0.649 & 0.719 & 0.721 \\
$\infty$ & 0.662 & 0.722 & 0.724 \\
\hline
\end{tabular}

the intra-population variance. However, this result of decreased equilibrium variance with decreased recombination could well be due to the gametic selection resorted to in the simulations.

\section{DISCUSSION}

Kimura (1965) showed, for infinitely large populations, and assuming a continuous time process that the distribution of allelic effects tends to be normal at equilibrium between selection and mutational forces and that the mean and variance of the equilibrium distribution are determined by the amounts of increase in mean and variance of the genotypic value per gene per generation as well as by the intensity of fitness function. The discrete allelic-state model with the assumption of a discrete-time process, considered in this paper, has revealed behaviour similar to those to Kimura (1965) as it should, since binomial distribution of allelic effects should tend to normal distribution as we go from discrete to continuous case. On the other hand, the diallelic model of Latter (1960), re-analysed by Bulmer (1972, 1980) who used different approximations but arrived at the same conclusions as those of Latter (1960), indicated different results. Their predictions for equilibrium genetic variance differ qualitatively from those of Kimura-Lande-Fleming-Narain \& Chakraborty.

Based on "House-of-cards" approximation of Kingman (1978), Turelli (1984) presented a new asymptotic analysis of Kimura's model to show that the qualitatively different predictions about equilibrium genetic variance are not due to the number of alleles assumed per locus. Instead, such different results are attributable to assumptions concerning the relative magnitudes of per locus mutation rates, the phenotypic effects of mutation 
and intensity of selection. He then analysed a model with tri-allelic loci which allows amonglocus variation in mutation rates and allelic effects. At the single locus level, such a model is a particular case of the discrete-time, discrete allelicstate mutation model analysed in the present paper and leads to the same conclusions as in Turelli (1984) in so far as the equilibrium genetic variance is concerned. This is demonstrated below.

For this purpose, we consider $m=1$ and a finite number of $(2 k+1)$ alleles $A_{-k}, A_{-(k-1)}, \ldots A_{-1}$, $A_{0}, A_{1}, \ldots, A_{(k-1)}, A_{k}$ at the locus following the mutation scheme given by (1) and note that $s$ in (3) corresponds to $\left(2 V_{s}\right)^{-1}$ in Turelli (1984). Under the same approximations for $v$ and $s$, as adopted in this study, but with finite number of $(2 k+1)$ alleles, the change in the frequency of $i$ th allele given by (A2) in Appendix A gets modified to

$$
\begin{aligned}
\Delta x_{i}(t)= & -v\left[x_{i}(t)\left\{1-\frac{x_{k}(t)+x_{-k}(t)}{2}\right\}\right. \\
& \left.-\frac{x_{i+1}(t)+x_{i-1}(t)}{2}\right] \\
& +s x_{i}(t)\left[\sum_{j=-k}^{k} a^{2} j^{2} x_{j}(t)\right. \\
& +2\left(\sum_{j=-k}^{k} a j x_{j}(t)\right)^{2}-a^{2} i^{2} \\
& \left.-2 a i \sum_{j=-k}^{k} \operatorname{ajx}_{j}(t)\right]
\end{aligned}
$$

When the population reaches globally stable equilibrium, $\Delta x_{i}(t)=0$ giving $\hat{x}_{i}=\hat{x}_{-i}$ for each $i$, the equilibrium mean is zero and we have, with $\hat{x}_{0}+2 \sum_{i=1}^{k} \hat{x}_{i}=1$,

$$
\begin{aligned}
& v\left[\hat{x}_{i}\left(1-\hat{x}_{k}\right)-\frac{\hat{x}_{i+1}+\hat{x}_{i-1}}{2}\right] \\
& =s \hat{x}_{i}\left[\sum_{j=-k}^{k} a^{2} j^{2} \hat{x}_{j}-a^{2} i^{2}\right], \\
& i=0,1,2, \ldots, k
\end{aligned}
$$

For $k=1$, we have three alleles $A_{0}, A_{1}$ and $A_{-1}$ with respective equilibrium frequencies $\hat{x}_{0}, \hat{x}_{1}=$ $\hat{x}_{-1}$ so that in view of $\hat{x}_{0}+2 \hat{x}_{1}=1$, we have only one unknown say $\hat{x}_{1}$ to be determined from the above equations. This leads to the quadratic equation in $\hat{x}_{1}$ as

$$
2(1+\beta) \hat{x}_{1}^{2}-(1+4 \beta) \hat{x}_{1}+\beta=0
$$

where $\beta=\left(v / 2 s a^{2}\right)$. For $\beta<1$, which corresponds to the condition (3.27) in Turelli (1984), we have approximately one permissible root as

$$
\hat{x}_{1}=\beta=v /\left(2 s a^{2}\right)
$$

The equilibrium genetic variance at the tri-allelic locus can then be shown to be equal to

$$
\begin{aligned}
\hat{\sigma}_{g_{A}}^{2} & =2 a^{2}\left[\hat{x}_{0}\left(1-\hat{x}_{0}\right)+4 \hat{x}_{1} \hat{x}_{-1}\right] \\
& =4 a^{2} \hat{x}_{1} \\
& =(2 v / s)
\end{aligned}
$$

which for $n$ loci, under the assumption of approximate global linkage equilibrium, would correspond exactly to the relation (3.29) of Turelli (1984). The results of this particular case therefore agrees with 'house-of-cards' predicition that equilibrium genetic variance is independent of the phenotypic effects of mutation to first order. It further indicates that the approximation $\beta<1$ which is true for a wide range of parameter values, as per Turelli (1984), is consistent with some of the numerically chosen values of $v$ and $s$ used in the present paper and therefore make the numerical results biologically relevant. However, it may be noted that as soon as we move from a finite number of alleles flanking on either side of the type allele $A_{0}$, to an infinitely large number of alleles similarly placed, we find that the equilibrium genetic variance is related to the fourth moment of the genotypic values in the manner given by (17). This indicates the importance of the non-normality of the distribution of genetic effects.

As already mentioned in the Introduction, very few studies on the problem of genetic differentiation between population or species have appeared, particularly for the situation when one of the daughter populations has a shifted optimum. Such cases have biological relevance as for instance in skin pigmentation for a small group of Caucasian race with fair skin who moved out of Central Asia around 3000 years ago and settled in southern parts of America with plenty of sunlight. The optimum phenotype for skin pigmentation must therefore have shifted by several standard deviations away from the original optimum. This introduces differentiation between populations and it is of interest to study the transient properties of such a process. This has been done in this paper by studying the distribution of allelic frequency as a function of time. Significant changes in statistical properties of the distribution such as mean, variance, skewness and kurtosis have been noticed. In particular, algebraic expressions for changes in mean and variance reveal interesting results. When we consider optimum at $d$ standard deviation units away from the origin, the mean change in the 
genotypic value of the character at the $t$ th generation, as given by eq. (15), depends on $s, d, \sigma_{p}$, $\mu_{3}(t-1), \mu_{2}(t-1)$ and $\mu_{1}^{\prime}(t-1)$. It does not depend on the mutation rate, $v$. But the change in the genotypic variance per generation, as given by eq. (16), clearly indicates that it is affected by the mutational component. Initially, the population is in equilibrium and symmetrical. If we further assume that it has normal kurtosis, we have $\mu^{\prime}(0)=$ $0, \mu_{3}(0)=0, \mu_{4}(0)=3 \mu_{2}^{2}(0)$. If we also disregard the increase in variation due to mutation, the expression (16) reduces to the result given in Latter (1970). The genotypic variance is reduced and the reduction depends on the intensity of selection and the heritability.

The transient behaviour of the mean noticed in this investigation can be useful in giving some idea about the divergence time; at least the time by which the mean gets to half of the total change in the mean, i.e., $1 / 2\left(d \sigma_{p}\right)$, which is known. The computer results show that intense selection speeds up the divergence time but by increasing the number of mutational steps, this time is shortened, though not very appreciably. Thus, for $v=0.001$, $s=0.008$ and $m=1$, this time is of the order of 120 generations. With $s=0.002$ and $m=1$ and 5 , the times are respectively 300 and 170 generations.

The main focus of this study is on the genetic differentiation between populations which gets built up over time when the population, after reaching equilibrium by mutation-selection balance, splits up into two in one of which the same optimum holds but in the other it shifts a few standard deviations away from the mean. The ratio between versus within population variance $\left(B_{t} / V_{t}\right)$ as a function of time then provides with a possible means of ascertaining the role of adaptive changes under which the character changes over time. With selection, this ratio necessarily changes nonlinearly with time.

Acknowledgements We are grateful to Professor M. Nei for suggesting this problem to us and for his continued interest and encouragement during the various phases of the work. The financial support of this research was provided by US Public Health Service research grants GM 28574 and GM 20293 from the National Institute of Health.

\section{REFERENCES}

BULMER, M. (j. 1972. The genetic variability of polygenic characters under optimizing selection of mutation and drift. Genet. Res. 19, 17-25.

BULMER, M. (i. 1980. The Mathematical Theory of Quantitative Genetics. Clarendon Press, Oxford.
(AVAI.II-SFORT.A, L. L. ANI) FHLDMAN, M. W. 1976. Evolution of continuous variation: direct approach through joint distribution of genotypes and phenotypes. Proc. Natn. Acad. Sci. USA, 73, 1689-1692.

(HAKRABORTY, R. AND NEI, M. 1982. Genetic differentiation of quantitative characters between populations or species I. Mutation and random genetic drift. Genet. Res., 39, 303-314

CLAYTON, (i. A. ANI) ROBERTSON, A. 1955. Mutation and quantitative variation. Am. Nat., 89, 151-158.

( ROW, J. F. ANI) KIMURA, M. 1964. The theory of genetic loads. Proc: XI Int. Congr. Genet., 495-505.

FALCONER, D. S. 1960. An Introduction to Quantitative Genetics. Oliver and Boyd, Edinburgh

Fishre, R. A. 1922. On the dominance ratio. Proc. Roy. Soc. Edin., 42, 321-341.

FLEMING, W. H. 1979. Equilibrium distributions of continuous polygenic traits. SIAM J. Appl. Math., 36, 148-168.

GIILESPIE, J. H. 1984. Pleiotropic overdominance and the maintenance of genetic variation in polygenic characters. Genetics, 107, 321-330.

GIMHLFARB, A. 1986. Additive variation maintained under stabilizing selection: A two-locus model of pleiotropy for two quantitative characters. Genetics, 112, 717725.

HALIJANE, J. B. S. 1932. The Causes of Evolution, Longmans, London.

HALDANF, J. B. S. 1954. The measurement of natural selection Proc: 9th Internl. Cong. Genet., 1, 480-487.

KARL.IN, S. 1979. Models of multifactorial inheritance. I-IV. Theor. Pop. Biol., 15, 308-438.

KIMURA, M. 1965. A stochastic model concerning the maintenance of genetic variability in quantitative characters. Proc Natl. Acad. Sci. U.S., 54, 731-736.

KINCiMAN, I. F. C. 1978. A simple model for the balance between selection and mutation. J. appl. Pro., 15, 1-12.

LANDE, R. 1975. The maintenance of genetic variability by mutation in a polygenic character with linked loci. Genet. Res., Camb., 26, 221-235.

LANDE, R. 1976. Natural selection and random genetic drift in phenotypic evolution. Evolution, 30, 314-334.

I.ANDE, R. 1977. The influence of the mating system on the maintenance of genetic variability in polygenic characters. Genetics, 86, 485-498

LANDE, R. 1979. Quantitative genetic analysis of multivariate evolution applied to brain: body size allometry. Evolution, 33, 402-416.

LANDE, $R, 1980$. The genetic covariance between characters maintained by pleiotropic mutations. Genetics, 94, 203215 .

IATTER, B. 1). H. 1960. Natural selection for an intermediate optimum. Aust. J. Biol. Sci., 13, 30-35.

LAITER, B. D. H. 1970. Selection in finite populations with multiple alleles. II. Centripetal selection, mutation and isoallelic variation. Genetics, 66, 165-186.

LATTER, B. D. H. ANI) N(JVI'TSKI, C. E. 1969. Selection in finite populations with multiple alleles. I. Limits to directional selection. Genetics, 62, 859-876.

NAGYLAKI, T; 1984. Selection on a quantitative character. Chakravarti, A. (ed.) In Human Population Genetics: The Pittshurgh Sympo. Hutchinson Ross. Stroudsburgh Pa., 275-306.

ROBERTSON, A. 1956. The effect of selection against extreme deviants based on deviation or on homozygosis. J. Genet., $54,236-248$

SLATKIN, M. 1970. Selection and polygenic characters. Proc. Natn. Acad. Sci. USA, 66, 87-93. 
SUZUKI, H. 1960. Temporal changes of anthropometric characters of Japanese. Oka, H. (ed.) In: Proceedings of the Darwin Centennial Symp. on Evolution 140-149. Tokyo: Japan Society for the Promotion of Science.

TURELLI, M. 1984. Heritable Genetic Variation via MutationSelection balance: Lerch's Zeta meets the Abdominal Bristle. Theo. Pop. Biol., 25, 138-193.

TURELLI, M. 1985. Effects of pleiotropy on predictions concerning mutation-selection balance for polygenic traits. Genetics, 111, 165-195.

WRIGHT, S. 1931. Evolution in Mendelian populations. Genetics, 16, 97-159.

WRIGHT, S. 1937. The distribution of gene frequencies in populations. Proc. Natl. Acad. Sci. U.S., 23, 307-320.

\section{APPENDIX A}

\section{Allele frequency profile in an equilibrium population under optimum selection with one step mutation model}

With a one step discrete allelic state mutation model, the recurrence equation (4) reduces to

$$
\begin{aligned}
x_{i}(t)= & \frac{v}{2}\left[x_{i+1}(t-1)+x_{i-1}(t-1)\right] \\
& +x_{i}(t-1)\left[1-v-s\left\{a^{2} i^{2}-\sigma_{g_{A}}^{2}(t-1) / 2\right\}\right]
\end{aligned}
$$

and thus the change in the frequency of the $i$ th allele per generation becomes

$$
\begin{aligned}
\Delta x_{i}(t)= & -v\left[x_{i}(t)-\frac{x_{i+1}(t)+x_{i-1}(t)}{2}\right] \\
& +s x_{i}(t)\left[\frac{\sigma_{g_{A}}^{2}(t)}{2}-a^{2} i^{2}\right]
\end{aligned}
$$

When the population reaches equilibrium under the opposing pressures of mutation and selection, we have $\Delta x_{i}=0$ to get

$$
\hat{x}_{1}=[1-S G] \hat{x}_{0}
$$

and

$$
x_{i+1}-2\left[1-S\left(G-i^{2}\right)\right] \hat{x}_{i}+\hat{x}_{i-1}=0 \text { for } i \geq 1
$$

with $\hat{x}_{i}=\hat{x}_{-i}$ for all non-zero integers $i$.

In (A3) $S$ and $G$ are the same as defined in eq. (6). It is apparent that (A3) is a second order linear difference equation with variable coefficients and can be solved by standard technique of transforming (A3) into

$$
\Delta^{2} \hat{x}_{i}+2 S\left[G-(i+1)^{2}\right] \Delta \hat{x}_{i}+2 S\left[G-(i+1)^{2}\right] \hat{x}_{i}=0 .
$$

Substituting $x_{i}=\sum_{k=0}^{\infty} C_{k} i^{(k)}$ into (A4) where the factorial function $i^{(k)}$ is defined by $i^{(k)}=$ $i(i-1) \ldots(i-k+1)$, we get a polynomial equation in $i$, which in turn is an identity, so that each coefficient of $i^{k}$ becomes zero. This yields

$$
\begin{aligned}
& 2 C_{2}+ 2 S\left(G-1^{2}\right) C_{1}+2 S(G-1 \cdot 1) C_{0}=0 \\
& 6 C_{3}+4 S\left(G-2^{2}\right) C_{2}+2 S(G-3 \cdot 2) C_{1}-6 S C_{0}=0 \\
& 12 C_{4}+6 S\left(G-3^{2}\right)+2 S(G-5 \cdot 3) C_{2} \\
& \quad-10 S C_{1}-2 S C_{0}=0 \\
& 20 C_{5}+8 S\left(G-4^{2}\right) C_{4}+2 S(G-7 \cdot 4) C_{3} \\
&-14 S C_{2}-2 S C_{1}=0
\end{aligned}
$$

etc.

Two arbitrary solutions for $\hat{x}_{i}$ are obtained as $\hat{x}_{i 1}$ and $\hat{x}_{i 2}$ by substituting $C_{0}=0, C_{1}=1$ and $C_{0}=1$, $C_{1}=0$, respectively, to get

$$
\begin{aligned}
\hat{x}_{i 1}= & i^{(1)}+S(1-G) i^{(2)} \\
& +\frac{S}{3}[(6-G)+2 S(1-G)(4-G)] i^{(3)} \\
& +\frac{S}{6}[5+S\{(1-G)(15-G)+(9-G)(6-G)\} \\
& \left.+2 S^{2}(1-G)(4-G)(9-G)\right] i^{(4)} \\
& +\ldots
\end{aligned}
$$

and

$$
\begin{aligned}
\hat{x}_{i 2}= & +S(1-G) i^{(2)} \\
& +\frac{S}{3}[3+2 S(1-G)(4-G)] i^{(3)} \\
& +\frac{S}{6}[1+S\{(1-G)(15-G)+3(9-G)\} \\
& \left.+2 S^{2}(1-G)(4-G)(9-G)\right] i^{(4)} \\
& +\ldots
\end{aligned}
$$

The general solution is then

$$
\hat{x}_{i}=\alpha_{1} \hat{x}_{i 1}+\alpha_{2} \hat{x}_{i 2} .
$$

For $i=0, \hat{x}_{01}=0$ and $\hat{x}_{02}=1$, so that

$$
\alpha_{2}=\hat{x}_{0} \text {. }
$$

For $i=1, \hat{x}_{11}=1$ and $\hat{x}_{12}=1$, so that $\hat{x}_{1}=\alpha_{1}+\hat{x}_{0}$. Using (A3) we get

$$
\alpha_{1}=-(S G) \hat{x}_{0} .
$$

Thus we get

$$
\hat{x}_{1}=\left[\hat{x}_{i 2}-(S G) \hat{x}_{i 1}\right] \hat{x}_{0}
$$


where $x_{i 1}$ and $x_{i 2}$ are given as above. From (A5) we see, putting $i=1$, that $S G<1$ which means

$$
\hat{\sigma}_{g_{A}}^{2}<(2 v / s) \text {, }
$$

giving the upper limit of the equilibrium variance in terms of $v$ and $s$. This was found to be true from the computer results using the exact recurrence relation (4) for $m=1$. The final form for $\hat{x}_{i}$, using (A5) in conjunction with the specific solutions $\hat{x}_{i 1}$ and $\hat{x}_{i 2}$ is obtained as

$$
\begin{aligned}
\hat{x}_{i}= & \hat{x}_{0}\left\{1-S G i^{(1)}+S(1-G)(1-S G) i^{(2)}\right. \\
& +\frac{S}{3}[3-S G(6-G) \\
& +2 S(1-G)(4-G)(1-S G)] i^{(3)} \\
& +\frac{S}{6}[1-5 S G+S\{(1-G)(15-G)(1-S G)
\end{aligned}
$$

$$
\begin{aligned}
& +3(9-G)(1-S G(6-G))\} \\
& \left.+2 S^{2}(1-G)(4-G)(9-G)(1-S G)\right] i^{(4)} \\
& +\ldots\}
\end{aligned}
$$

Numerically, for all practical purposes, we find that the frequencies of alleles at equilibrium are virtually zero beyond $\hat{x}_{3}$. We can therefore obtain $\hat{x}_{1}, \hat{x}_{2}$ and $\hat{x}_{3}$ from (A6) as follows

$$
\begin{aligned}
\hat{x}_{1}= & \hat{x}_{0}[1-S G] \\
\hat{x}_{2}= & \hat{x}_{0}[1-2 S G+2 S(1-G)(1-S G)] \\
\hat{x}_{3}= & \hat{x}_{0}[1-3 S G+6 S(1-G)(1-S G) \\
& +2 S\{3-S G(6-G) \\
& +2 S(1-G)(4-G)(1-S G)\}]
\end{aligned}
$$

which are the same as in eq. (6). 